Pacific Journal of Mathematic 


\title{
THE MULTIPLIER ALGEBRA OF A CONVOLUTION MEASURE ALGEBRA
}

\author{
KARI YLINEN
}

\begin{abstract}
In this paper the structure theory of convolution measure algebras due to J. L. Taylor is used in studying the multiplier algebra $M(A)$ of a commutative semi-simple convolution measure algebra $A$. A criterion is given for the embeddability of $M(A)$ in the measure algebra $M(S)$ on the structure semigroup $S$ of $A$, and the relationship between the structure semigroups of $A$ and $M(A)$ is investigated in case $M(A)$ is also a convolution measure algebra and $S$ has an identity.
\end{abstract}

1. Introduction. A convolution measure algebra $A$ is a complex $L$-space with a multiplication which gives $A$ the structure of a Banach algebra and satisfies certain additional requirements. For precise definitions and the basic theory of convolution measure algebras we refer to J. L. Taylor's paper [11]. A central role in Taylor's theory is played by the structure semigroup $S$ of a commutative convolution measure algebra $A$. The maximal regular ideal space of $A$ may be identified with the set of semicharacters of the compact commutative topological semigroup $S$, and some properties of $A$ are reflected in those of $S$.

For any (complex) commutative Banach algebra $A$, let $\Delta(A)$ denote the spectrum of $A$, that is, the space of nonzero multiplicative linear functionals on $A$, equipped as usual with the relative weak topology. If $A$ is in addition semisimple, then we denote by $A^{m}$ the space of all complex-valued functions on $\Delta(A)$ that keep the space $\hat{A}$ of the Gelfand transforms $\hat{x}$ of the elements $x$ of $A$ invariant by pointwise multiplication, i.e., $A^{m}=\{f: \Delta(A) \rightarrow C \mid f \hat{x} \in \hat{A}$ for all $x \in A\}$. It can be easily shown that each $f \in A^{m}$ determines a unique bounded linear operator $T_{f}: A \rightarrow A$ satisfying $\widehat{T_{f} x}=f \hat{x}, x \in A$. Then $M(A)=\left\{T_{f} \mid f \in A^{m}\right\}$ is a Banach algebra under the uniform operator norm, called the multiplier algebra of $A$. For the general theory of multiplier algebras one may consult e.g. Larsen's book [5].

In this paper we study the multiplier algebra of a commutative semi-simple convolution measure algebra $A$. J. L. Taylor has shown in [11] that $A$ may be naturally embedded in the convolution algebra $M(S)$ of finite regular Borel measures on the structure semigroup $S$. In $\S 3$ we show that $M(A)$ can be isometrically realized as a subalgebra of $M(S)$ containing the image of $A$ if and only if $S$ has an identity. As is to be expected, the measures then corresponding to isometric onto multipliers have one point support in $S$. Section 4 gives con- 
ditions for $M(A)$ to be a convolution measure algebra, too, and $\S 5$ concentrates on describing the relationship that exists between $S$ and the structure semigroup of $M(A)$ provided $M(A)$ is a convolution measure algebra and $S$ has an identity. For related results in a somewhat different situation, see [13].

For any compact Hausdorff space $S, C(S)$ will denote the Banach space of continuous complex-valued functions on $S$ with the supremum norm, and $M(S)$ is the conjugate space of $C(S)$. Of course, $M(S)$ may be interpreted as the space of finite regular Borel measures on $S$, and if $S$ is also a topological semigroup, $M(S)$ is a Banach algebra under the convolution product

$$
\mu * \nu(f)=\int_{S} \int_{S} f(x y) d \mu(x) d \nu(y) .
$$

2. Taylor's structure semigroup of a commutative convolution measure algebra. Preliminarily to our discussion of the multiplier algebra we give in this section the structure semigroup a description which differs slightly from Taylor's original construction. In special cases an essentially similar method has been used e.g. by Rennison in [8] and Ramirez in [7]. See also [6] and [13].

The conjugate space $A^{\prime}$ of any complex $L$-space $A$ is a commutative $C^{*}$-algebra with identity. The corollary in [11, p. 157] says that if $A$ is a commutative convolution measure algebra, then $\Delta(A) \cup\{0\}$ is a self-adjoint multiplicative subsemigroup of $A^{\prime}$ containing the identity, so that the norm closed linear span $P$ of $\Delta(A)$ in $A^{\prime}$ is a $C^{*}$-algebra with identity. A semicharacter on a topological semigroup is a non-zero continuous homomorphism into the multiplicative semigroup of complex numbers $z$ with $|z| \leqq 1$.

THEOREM 2.1. Let $A$ be a commutative convolution measure algebra and $P$ as above. For any $F, G \in P^{\prime}$ there is a unique element, denoted $F G$, of $P^{\prime}$ such that $F G(\alpha)=F(\alpha) G(\alpha)$ for all $\alpha \in \Delta(A)$. The map $(F, G) \mapsto F G$ is a commutative Banach algebra product in $P^{\prime}$. The spectrum $\Delta(P)$ of $P$ is a multiplicative subsemigroup of $P^{\prime}$. With the relative weak* topology $\Delta(P)$ is a compact topological semigroup, and the semicharacters of $\Delta(P)$ are precisely the Gelfand transforms of the elements of $\Delta(A)$. The structure semigroup $S$ of $A$ in the sense of Taylor [11] is topologically isomorphic to $A(P)$.

Proof. The product in $P^{\prime}$ that we are referring to is discussed in [1, p. 816] and [13, pp. 168-169]. In particular, since $F G(\alpha \beta)=$ $F(\alpha \beta) G(\alpha \beta)$ for all $\alpha, \beta \in \Delta(A), F, G \in P^{\prime}$, even if $\alpha \beta=0$, the proof of Theorem 2.3 in [13] is valid also in our present situation where, 
in general, merely $\Delta(A) \cup\{0\}$ is a multiplicative subsemigroup of $A^{\prime}$. Similarly, Theorem 2.4 in [13] is applicable, for the semi-simplicity of $A$ is nowhere needed in its proof, and $\Delta(A)$ (rather than $\Delta(A) \cup\{0\}$ ) is assumed to be closed with respect to multiplication only to allow one to appeal to the above mentioned Theorem 2.3. From the proof of Theorem 2.2 in [11] it is clear that there is a homeomorphism $\varphi$ from the structure semigroup $S$ of $A$ onto $A(P)$ such that its natural dual map from $C(\Delta(P))$ onto $C(S)$ puts the sets of semicharacters on $S$ and $\Delta(P)$ in a bijective correspondence. As in the proof of Theorem 6.5 in [7] it is seen that $\phi$ is also a semigroup isomorphism.

From now on we call $S(P)$ with the product mentioned in the above theorem the structure semigroup of $A$ and use the notation $S=\Delta(P)$.

Theorem 2.2. Let $A$ and $P$ be as in Theorem 2.1. If $P^{\prime}$ is given the product referred to in that theorem, then the isometric embedding $F \mapsto \mu_{F}$ from $P^{\prime}$ onto $M(S)=C(S)^{\prime}$ defined by $\left\langle f, \mu_{F}\right\rangle=$ $\langle f, F\rangle$ for $F \in P^{\prime}, f \in P=C(S)$, is an algebra isomorphism.

Proof. Suppose $F, G \in P^{\prime}$. By the definition of the convolution $\mu_{F} * \mu_{G}$ we have for any $\alpha \in \Delta(A)$,

$$
\begin{aligned}
\left\langle\hat{\alpha}, \mu_{F} * \mu_{G}\right\rangle & =\int_{S} \int_{S} \hat{\alpha}(x y) d \mu_{F}(x) d \mu_{G}(y)=\int_{S} \hat{\alpha}(x) d \mu_{F}(x) \int_{S} \hat{\alpha}(y) d \mu_{G} \\
& =\langle\alpha, F\rangle\langle\alpha, G\rangle=\langle\alpha, F G\rangle=\left\langle\hat{\alpha}, \mu_{F G}\right\rangle .
\end{aligned}
$$

Since the functions $\hat{\alpha}, \alpha \in \Delta(A)$, generate the Banach space $C(S)$, the equality $\left\langle h, \mu_{F} * \mu_{G}\right\rangle=\left\langle h, \mu_{F G}\right\rangle$ is valid for all $h \in C(S)$, i.e., $\mu_{F} * \mu_{G}=\mu_{F G}$.

3. Representing the multipliers as measures on the structure semigroup. Throughout the rest of the paper we assume that $A$ is a commutative, semi-simple convolution measure algebra with structure semigroup $S=\Delta(P)$, where $P$ is always the closed linear span of $A(A)$ in $A^{\prime}$. The set of semicharacters on $S$ is denoted by $\widehat{S}$. We give $P^{\prime}$ the Banach algebra product mentioned in Theorem 2.1.

LEMma 3.1. The natural embedding $0: A \rightarrow P^{\prime}$ defined by $\langle f, \theta x\rangle=$ $\langle x, f\rangle, x \in A, f \in P$, is an isometric and bipositive (i.e., $\theta x \geqq 0$ if and only if $x \geqq 0$ ) algebra homomorphism.

Proof. From the definition of the product in $P^{\prime}$ it is clear that $\theta$ is a homomorphism. Theorem 2.3 in [11] along with the corollary in [11, p. 154] shows that it is isometric and bipositive. Alternatively, $\theta$ is isometric by virtue of the Kaplansky density theorem [10, p. 22], and bipositive by Propositions 1.5.1 and 1.5.2 in [10, p. 9], since $P$ 
contains the identity of $A^{\prime}$.

We usually identify $P^{\prime}$ and $M(S)$ as ordered Banach spaces and Banach algebras in accordance with Theorem 2.2. Following Taylor [11], we use the notation $\theta(A)=A_{S} \subset M(S)$. It follows e.g. from [11, Theorem 2.3] and the corollary in [11, p. 154] that $A_{S}$ is an $L$-subspace [11, p. 151] of the complex $L$-space $M(S)$.

Lemma 3.2. The convolution product in $M(S)$ is separately weak* continuous.

Proof. Suppose $\nu \in M(S)$ and $f \in C(S)$. It is a simple matter to show that the function $\varphi, \varphi(y)=\int_{S} f(x y) d \nu(x)$, is continuous on $S$. (A much more general result may be proved using Grothendieck's weak compactness theorem, see [3, p. 205].) Since we have $|\langle f, \nu * \mu\rangle|=$ $\left|\int_{S} \int_{S} f(x y) d \nu(x) d \mu(y)\right|=|\langle\varphi, \mu\rangle|$, the mapping $\mu \mapsto \nu * \mu$ is weak* continuous at zero, hence everywhere.

Lemma 3.3. Suppose that $S$ has an identity and $\mu \in M(S)$. Then $\mu \geqq 0$ if and only if $\mu * \nu \geqq 0$ for all $\nu \geqq 0$ in $A_{s}$.

Proof. Clearly the latter condition is necessary. Suppose now that $\mu * \nu \geqq 0$ for all $\nu \geqq 0$ in $A_{s}$. Choose a basis $\mathscr{Q}$ of compact neighborhoods of the identity $u$ of $S$, directed in the natural order opposite to inclusion. Each $\nu \in A_{S}$ is a linear combination of nonnegative elements of $A_{S}$ and if $\lambda \in M(S), 0 \leqq \lambda \leqq \nu \in A_{S}$, then $\lambda \in A_{S}$ since $A_{S}$ is an $L$-subspace of $M(S)$. Furthermore, since $A_{S}$ separates $P=C(S), A_{S}$ is a weak* dense subspace of $M(S)$. It follows easily that for each $U \in \mathscr{U}$ there exists a positive measure $\mu_{U} \in A_{S}$, $\left\|\mu_{S}\right\|=1$, with support contained in $U$. The net $\left(\mu_{U}\right)_{U \in \mathscr{U}}$ converges to the Dirac measure $\delta_{u}$ in the weak* topology. By assumption, $\mu_{*} \mu_{U} \geqq 0$ for all $U \in \mathscr{U}$, and since the positive cone in $M(S)$ is weak* closed and the convolution is separately weak* continuous (Lemma 3.2 ), it follows that $\mu=\mu * \delta_{u}=\lim _{U} \mu * \mu_{U} \geqq 0$.

We regard the multiplier algebra $M(A)$ as an ordered Banach space with positive cone $\{T \in M(A) \mid T x \geqq 0$ for all $x \geqq 0$ in $A\}$.

THEOREM 3.1. If $S$ has an identity, then there exists a bipositive, isometric algebra isomorphism from $M(A)$ onto the subalgebra $B=$ $\left\{\mu \in M(S) \mid \mu * \nu \in A_{S}\right.$ for all $\left.\nu \in A_{S}\right\}$ of $M(S)$. Conversely, if there exists an isometric algebra isomorphism $\psi$ from $M(A)$ onto a subalgebra of $M(S)$ containing $A_{S}$, then $S$ has an identity, and for any isometric and surjective $T \in M(A)$ we have $\psi(T)=c \delta_{x}$, where $c \in C,|c|=1$ and $\delta_{x}$ is the Dirac measure corresponding to some $x \in S$. 
Proof. Suppose that $S$ has an identity $u$. The net $\left(\mu_{U}\right)_{U \in \mathscr{Z}}$ constructed in the proof of Lemma 3.3 converges to the Dirac measure $\delta_{u}$ in the weak* topology of $M(S)$. In particular, $\lim _{U}\left\langle\mu_{U}, \gamma\right\rangle=1$ for each $\gamma \in \Delta(A)=\widehat{S}$. If we denote by $T_{f} \in M(A)$ the operator corresponding to the function $f \in A^{m}$ (see the introduction), an argument given in [1, p. 817] shows that $\left|\sum_{k=1}^{n} a_{k} f\left(\gamma_{k}\right)\right| \leqq\left\|T_{f}\right\|\left\|\sum_{k=1}^{n} a_{k} \gamma_{k}\right\|$ for all $\gamma_{k} \in \Delta(A), a_{k} \in C, k=1, \cdots, n$. It follows that $f$ can be extended as a continuous linear functional $f$ to the whole of $P$ with norm $\|\boldsymbol{f}\| \leqq\left\|T_{f}\right\|$. Since the embedding $\theta: A \rightarrow P^{\prime}$ is isometric (Lemma 3.1 , we have, using the definition of the product in $P^{\prime},\|\boldsymbol{f}\| \geqq$ $\sup \{\|\boldsymbol{f} \theta(x)\| \mid x \in A,\|\theta(x)\| \leqq 1\}=\sup \{\|\boldsymbol{f} \theta(x)\| \mid x \in A,\|x\| \leqq 1\}=$ $\sup _{\substack{\|x\| \leq 1 \\ x \in A}}\left\|T_{f} x\right\|=\left\|T_{f}\right\|$. Thus $\|\boldsymbol{f}\|=\left\|T_{f}\right\|$. From the definition of the product in $P^{\prime}$ it is obvious that the above embedding of $M(A)$ in $P^{\prime}$ is an algebra homomorphism, so that it may be interpreted as an isometric algebra homomorphism $\pi: M(A) \rightarrow M(S)$ (Theorem 2.2). Since the embedding of $A$ in $M(S)$ is bipositive (Lemma 3.1), it is clear from Lemma 3.3 that $\pi$ is bipositive. Denote $\pi(M(A))=B \subset M(S)$. For functions in $A^{m}(\supset \widehat{A})$, pointwise multiplication corresponds to the convolution of the respective measures on $S$ (see the proof of Theorem 2.2). Therefore, $A_{S}$ is an ideal in $B$. Also, if $\mu \in M(S)$, and $\mu * \nu \in A_{S}$ for all $\nu \in A_{S}$, then the function $f_{\mu}: \Delta(A) \rightarrow C$ obtained by restricting $\mu$ to $\widehat{S}=\Delta(A)$ belongs to $A^{m}$. The first part of the theorem is thus proved. To prove the converse assertion we note that $M(A)$ has always an identity. The hypothesis then implies that a weak* dense subalgebra of $M(S)$ has an identity $\eta$. It follows from Lemma 3.2 that $\eta$ is also an identity for $M(S)$. But it is well known that the identity of any Banach algebra is an extreme point in its unit ball (see e.g. [10, p. 13]). Hence (see [2, p. 441]) we have $\eta=c \delta_{u}$ for some $u \in S$ and $c \in C,|c|=1$. In fact $c=1$, since $c \delta_{u}=c \hat{o}_{u} * c \hat{o}_{u}=$ $c^{2} \delta_{u^{2}}$, so that $u=u^{2}$ and $c=c^{2}$. Now, $u$ is an identity for $S$, since $\delta_{u x}=\delta_{u} * \delta_{x}=\delta_{x}$ for all $x \in S$. For the last statement, it is enough to show that $\psi(T)$ is an extreme point of the unit ball of $M(S)$ [2, p. 441]. If $0 \leqq \lambda \leqq 1$ and $\mu_{1}, \mu_{2} \in M(S)$ are such that $\psi(T)=\lambda \mu_{1}+$ $(1-\lambda) \mu_{2}$ and $\left\|\mu_{1}\right\| \leqq 1,\left\|\mu_{2}\right\| \leqq 1$, we have for the identity $\eta$ of $M(S)$, since also $T^{-1} \in M(A)$ [5, p. 15],

$$
\eta=\psi\left(T^{-1}\right) * \psi(T)=\lambda \psi\left(T^{-1}\right) * \mu_{1}+(1-\lambda) \psi\left(T^{-1}\right) * \mu_{2},
$$

where $\left\|\psi\left(T^{-1}\right) * \mu_{1}\right\| \leqq 1$ and $\left\|\psi^{\prime}\left(T^{-1}\right) * \mu_{2}\right\| \leqq 1$. Since $\eta$ is an extreme point of the unit ball of $M(S)$, we have $\lambda=0$ or $\lambda=1$. Therefore, $\psi(T)$ is also an extreme point of the unit ball of $M(S)$.

Note. From the proof of the above theorem it is clear that $S$ has an identity if and only if $A$ has a weak bounded approximate 
identity [1, p. 817] of norm one. (Compare [11, Theorem 3.1].)

4. $M(A)$ as a convolution measure algebra. If $S$ has an identity, then $M(A)$ may be embedded in $M(S)$ in accordance with Theorem 3.1. Unfortunately, the nature of the image $B \subset M(S)$ of $M(A)$ is not sufficiently clear on the basis of that result. For example, we should like to conclude that $B$ is a so-called $L$-subalgebra of $M(S)$, which turns out to be equivalent to saying that $M(A)$ with its natural order is a convolution measure algebra. The next theorem gives two other necessary and sufficient conditions for this to be case.

We assume henceforth that $S$ has an identity and let $\pi: M(A) \rightarrow$ $M(S)$ be the bipositive, isometric homomorphism constructed in the proof of Theorem 3.1, and denote as before $B=\pi(M(A))=\left\{\mu \in M(S) \mid \mu * \nu \in A_{S}\right.$ for all $\left.\nu \in A_{S}\right\}$. Since $S$ has an identity, $\Delta(A)$ (and not merely $\Delta(A) \cup\{0\}$ ) is a multiplicative subsemigroup of $A^{\prime}$, so that it makes sense to talk about translations of functions on $\Delta(A)$. A set $\mathscr{F}$ of functions $f: \Delta(A) \rightarrow C$ is translation invariant, if $f \in \mathscr{F}$ implies $f^{\alpha} \in \mathscr{F}$ for all $\alpha \in \Delta(A)$, where $f^{\alpha}(\beta)=f(\alpha \beta)$ for $\alpha, \beta \in \Delta(A)$.

A closed subalgebra $N$ of the convolution measure algebra $M(S)$ is an $L$-subalgebra of $M(S)$, if for any $\mu \in N$ its total variation $|\mu|$ belongs to $N$, and if $\nu \in N$ whenever $\mu \in N$ and $\nu$ is absolutely continuous with respect to $|\mu|$ (denoted $\nu \ll|\mu|$ ) [12, p. 257]. This definition is easily seen to be equivalent to requiring that $N$ be a subalgebra and an $L$-subspace of $M(S)$ in the sense of [11].

THEOREM 4.1. The following conditions are equivalent:

(i) $M(A)$ is a convolution measure algebra (in the order defined before Theorem 3.1),

(ii) $B$ is an L-subalgebra of $M(S)$,

(iii) $A^{m}$ is a translation invariant set of functions on $\Delta(A)$,

(iv) for any $\mu \in B,|\mu|$ also belongs to $B$.

Proof. We shall establish the following chain of implications: (ii) $\Rightarrow$ (iii) $\Rightarrow$ (iv) $\Rightarrow$ (ii) $\Rightarrow$ (i) $\Rightarrow$ (iv). If (ii) holds and $\mu \in B, f \in C(S)$, then the measure $f \mu$ (i.e., the functional $g \mapsto \mu(f g)$ on $C(S)$ ) belongs to $B$. But if $f \in A^{m}$ and $\mu_{f}=\pi\left(T_{f}\right)$, then we have $f^{\alpha}(\beta)=\mu_{f}(\alpha \beta)=\alpha \mu_{f}(\beta)$ for all $\alpha, \beta \in \Delta(A)=\hat{S}$, so that $f^{\alpha} \in A^{m}$, since $\alpha \mu_{f} \in B$ and $A_{S}$ is an ideal in $B$. Thus (ii) implies (iii). Next, assume (iii) and choose any $\mu \in B$. Then the function $f_{\mu}: \Delta(A) \rightarrow C$ defined by $f_{\mu}(\alpha)=\mu(\alpha)$ for $\alpha \in \Delta(A)=\widehat{S}$ belongs to $A^{m}$. By assumption, $\left(f_{\mu}\right)^{\alpha} \in A^{m}$ for any $\alpha \in \Delta(A)$. But the measure in $B$ corresponding to $\left(f_{\mu}\right)^{\alpha}$ when $\alpha$ is regarded as a semicharacter of $S$, is $\alpha \mu$. As $\widehat{S}$ generates the Banach space $C(S)$ and the mapping $f \mapsto f \mu$ is continuous from $C(S)$ to $M(S)$, which contains $B$ as a closed subspace, we have $f \mu \in B$ for all $f \in C(S)$. Fur- 
thermore, $C(S)$ is dense in $L^{1}(S,|\mu|)[4$, p. 140] so that there is a sequence of functions in $C(S)$ converging to $\bar{g}$ in $L^{1}(S,|\mu|)$, where $g$ is a $|\mu|$-measurable function with $|g(x)|=1|\mu|$-a.e. and such that $\mu=g|\mu|$ [4, p. 171]. By virtue of the continuity of the mapping $f \mapsto f \mu$ from $L^{1}(S,|\mu|)$ to $M(S)$ and the fact that $f \mu \in B$ for all $f \in C(S)$, it follows that $|\mu|=\bar{g} \mu \in B$, i.e., (iv) holds. Assume now (iv) and that $\mu \in M(S)$ is absolutely continuous with respect to some $\lambda \geqq 0$ in $B$. Then we have also $\mu_{j} \ll \lambda, j=1, \cdots, 4$, in the Jordan decomposition $\mu=\mu_{1}-\mu_{2}+i\left(\mu_{3}-\mu_{4}\right)$, where $\mu_{1}$ and $\mu_{2}$ (resp. $\mu_{3}$ and $\mu_{4}$ ) are mutually singular nonnegative measures. If $\nu \geqq 0$ is in $A_{s}$, we have $\nu * \mu_{j} \ll \nu * \lambda$. This has been proved in a somewhat more general setting by Pym in [6, p. 630]. Since $A_{S}$ is an $L$-subspace of $M(S)$, hence an $L$-subalgebra in the sense of [12], we have $\nu * \mu \in A_{S}$. It follows that in fact $\nu * \mu \in A_{S}$ for an arbitrary $\nu \in A_{S}$, so that $\mu \in B$. Thus (ii) holds. Since $M(A)$ is isometrically algebra and order isomorphic to $B$, and any $L$-subalgebra of $M(S)$ is a convolution measure algebra (see [11, p. 151 and Definition 2.1]), (ii) implies (i) at once. Finally, if $M(A)$ is a convolution measure algebra (hence a complex $L$-space), (iv) holds by virtue of Corollary 1.6 and Proposition 1.8 in [9].

On the basis of the above theorem sufficient conditions (assuming that $S$ has an identity) can be given to guarantee that $M(A)$ is a convolution measure algebra. Since $A_{S}$ is an $L$-subalgebra of $M(S)$, an argument used in the proof of the above theorem shows that $\hat{A}$ is translation invariant on $\Delta(A)$. If we assume for example that $A$ is regular and has a bounded approximate identity consisting of elements with Gelfand transforms of compact support, then the theorem in [1, p. 819] shows that $A^{m}$ consists of those functions on $\Delta(A)$ which belong locally to $\hat{A}$ and may be extended to continuous linear functionals on $P$. Then Theorem 4.2 in [13] shows that $A^{m}$ is translation invariant. Another case where the translation invariance of $A^{m}$ follows immediately from that of $\hat{A}$ arises, when $S$ is a multiplicative group, for then we have $\left(f^{\alpha} \hat{x}\right)(\beta)=f(\alpha \beta) \hat{x}^{\alpha^{-1}}(\alpha \beta)=\widehat{y}(\beta)$ for some $y \in A$, if $f \in A^{m}$ and $x \in A$. For a discussion of this kind of a situation, see [12].

5. The structure semigroups of $A$ and $M(A)$. We retain the general hypotheses and notational conventions made in $\S \S 3$ and 4 . In particular $S$ has an identity. Let us make the additional assumption that $M(A)$ is a convolution measure algebra (in the order defined before Theorem 3.1). When $A$ and $M(A)$ are realized as subalgebras of $M(S)$ (§3), it is seen that the embedding $j: A \rightarrow M(A)$ defined by $j(x)=T_{\hat{x}}$ is isometric and bipositive. It is readily seen to be an $L$ homomorphism [11, p. 152], since $A_{S}$ is an $L$-subspace of $M(S)$. We let $Q$ denote the closed linear span of $\Delta(M(A))$ in $M(A)^{\prime}$. Then $T=$ 
$\Delta(Q)$ with the usual topology and product is the structure semigroup of $M(A)$. Before stating Theorem 5.1, which relates $S$ and $T$ to each other, we prove an auxiliary result.

Lemma 5.1. The mapping $\Phi: P \rightarrow M(A)^{\prime}$ defined by $\langle\Phi f, L\rangle=$ $\langle\pi(L), f\rangle$ for $L \in M(A), f \in P=C(S)$, is an isometric $C^{*}$-algebra homomorphism which maps the identity of $P$ to that of $M(A)^{\prime}$, and we have

$$
j^{*} \circ \Phi(f)=f, \quad f \in P,
$$

for the transpose $j^{*}: M(A)^{\prime} \rightarrow A^{\prime}$ of $j . \quad$ Furthermore, $\Phi(P) \subset Q$.

Proof. Equation (1) is immediate. Since $j: A \rightarrow M(A)$ is an $L$ homomorphism, $j^{*}: M(A)^{\prime} \rightarrow A^{\prime}$ is a $C^{*}$-algebra homomorphism which preserves the identity [11, p. 153]. Therefore,

$$
j^{*}(\Phi \alpha \Phi \beta)=\alpha \beta \text { for } \alpha, \beta \in \Delta(A)=\hat{S} .
$$

As $S$ has an identity, $\alpha \beta \neq 0$. A simple calculation shows that since $\pi$ is a homomorphism, $\Phi \alpha$ and $\Phi \beta$ are multiplicative, so that by (2) their product is a multiplicative extension of $\alpha \beta$ to $M(A)$ (when $\alpha \beta$ is regarded as a functional on $j(A)$ ). Now, $\Phi(\alpha \beta)$ is also a multiplicative extension of $\alpha \beta$ to $M(A)$, and since there are only one of them [5, p. 24], we have $\Phi(\alpha \beta)=\Phi \alpha \Phi \beta$. A similar argument shows that $\Phi \mid \Delta(A)$ preserves involution. It follows that $\Phi$ is a $C^{*}$-algebra homomorphism. Since the identity $e_{1}$ of $A^{\prime}$ belongs to $\Delta(A)$ and the identity $e_{2}$ of $M(A)^{\prime}$ to $\Delta(M(A)$ ), the uniqueness of the multiplicative extension again shows that $\Phi e_{1}=e_{2}$. Since any $C^{*}$-algebra homomorphism is norm-decreasing $[10$, p. 5] it follows from (1) that $\Phi$ is isometric. The last statement is a consequence of the fact that $\Phi(\Delta(A)) \subset \Delta(M(A))$.

In the following theorem $\mathfrak{S}$ denotes the natural embedding of $M(A)$ in $M(T)$ [11, p. 158]. The identity map of a set $D$ is denoted by $i d_{D}$.

THEOREM 5.1. There exist unique continuous semigroup homomorphisms $\psi: S \rightarrow T$ and $\varphi: T \rightarrow S$ such that

(1) $\Phi f(t)=f \circ \varphi(t)$ and $\Psi g(s)=g \circ \psi(s)$ for all $t \in T, f \in C(S)=P, s \in S, g \in C(T)=Q$, where $\Psi=j^{*} \mid Q$ and $\Phi$ is the map defined in Lemma 5.1. Furthermore,

(a) $\varphi \circ \psi=i d_{S}$ and $\Psi \circ \Phi=i d_{P}$.

(b) $\psi(S)$ is a closed ideal in $T$.

(c) For the identity $u$ of $S$ we have $\psi \circ \varphi(t)=t \psi(u), t \in T$.

(d) If we denote $M_{S}(T)=\{\mu \in M(T)|| \mu \mid(T \backslash \psi(S))=0\}$, then $M_{S}(T)$ is an ideal in $M(T)$ and $\Psi^{*}(M(S))=M_{S}(T)$ for the transpose $\Psi^{*}$ : $M(S) \rightarrow M(T)$ of $\Psi$. 
(e) The diagram below commutes, and all maps appearing in it are algebra homomorphisms.

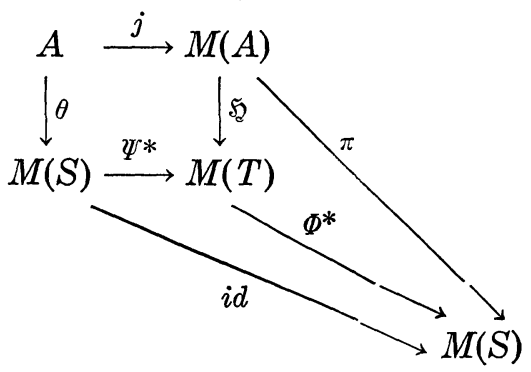

Proof. It is clear that (1) holds if and only if the maps $\psi: \Delta(P) \rightarrow$ $\Delta(Q)$ and $\varphi: \Delta(Q) \rightarrow \Delta(P)$ are defined by setting $\langle\psi(s), g\rangle=\Psi g(s)$ and $\langle\varphi(t), f\rangle=\Phi f(t)$ for $s \in S, t \in T, g \in Q=C(T)$, and $f \in P=C(S)$. From the definition of the product in $S$ and $T$ it follows that $\psi$ and $\varphi$, obviously continuous, are semigroup homomorphisms. For example, if $\gamma \in \Delta(M(A))$ and $x, y \in S$, then $\Psi \gamma \in \Delta(A)$ or $\Psi \gamma=0$, and in both cases $\langle\psi(x y), \gamma\rangle=\langle x y, \Psi \gamma\rangle=\langle x, \Psi \gamma\rangle\langle y, \Psi \gamma\rangle=\langle\psi(x), \gamma\rangle\langle\psi(y), \gamma\rangle$, i.e., $\psi(x y)=\psi(x) \psi(y)$. The second formula in (a) is a consequence of Lemma 5.1, and the first formula follows from the second by a simple calculation. The commutativity of the square in (e) is seen as follows: $\left\langle g, \Psi^{*} \circ \theta(x)\right\rangle=\langle\Psi g, \theta x\rangle=\langle x, \Psi g\rangle=\langle j(x), g\rangle=\langle g, \mathfrak{S} \circ j(x)\rangle$ for $x \in A$, $g \in C(T)=Q$, so that $\Psi^{*} \circ \theta=\mathfrak{S} \circ j$. The lower triangle commutes because of (a). As to the upper triangle, note that if $\gamma$ belongs to $\Delta(A)$, then $\Phi \gamma$ is its unique multiplicative extension to $M(A)$ (see the proof of Lemma 5.1). Therefore, $\left\langle\gamma, \Phi^{*} \circ \mathfrak{S}_{\mathcal{E}}(L)\right\rangle=\langle\Phi \gamma, \mathfrak{E}(L)\rangle=f_{L}(\gamma)=$ $\langle\gamma, \pi(L)\rangle$, where $f_{L}$ is the function in $A^{m}$ corresponding to $L \in M(A)$. Since $A(A)=\widehat{S}$ generates $C(S)$, the equation $\Phi^{*} \circ \mathfrak{S}=\pi$ follows. The second statement in (e) is also easily proved. Next we show that $\Psi^{*}(M(S))=M_{S}(T)$. Since $\Psi$ and $\Phi$ are norm-decreasing, $\Psi^{*}$ and $\Phi^{*}$ are so, and (e) implies that $\Psi^{*}$ is isometric. On the other hand, $\Psi^{*}$ is continuous from $\sigma(M(S), C(S))$ to $\sigma(M(T), C(T))$. Therefore, using the weak* compactness of $B_{r}=\{\mu \in M(S) \mid\|\mu\| \leqq r\}$, we see that $\left\{\mu \in \Psi^{*}(M(S)) \mid\|\mu\| \leqq r\right\}$ is weak ${ }^{*}$ compact, hence closed for each $r \geqq 0$. The Krein-Smulian theorem [2, p. 429] then shows that $\Psi^{*}(M(S))$ is weak* closed in $M(T)$. If $S$ (resp. $T$ ) is considered naturally embedded in $M(S)$ (resp. $M(T)$ ), then $\Psi^{*} \mid S=\psi$, so that $\Psi^{*}(S)=\psi(S)$. The linear combinations of the Dirac measures are weak* dense in $M(S)$. Similarly, since $\sigma\left(M_{S}(T), C(T)\right)$ and $\sigma\left(M_{S}(T), C(\psi(S))\right)$ coincide on $M_{S}(T)$, the linear span of $\psi(S)=\Psi^{*}(S)$ is $\sigma\left(M_{S}(T), C(T)\right)$-dense in $M_{S}(T)$, which in turn is weak* closed in $M(T)$, as $\psi(S) \subset T$ is compact. From these remarks the equation $\Psi^{*}(M(S))=M_{S}(T)$ follows by the weak* continuity of $\Psi^{*}$. From (e) and the fact that $j(A)$ is 
an ideal in $M(A)$ it follows that $\Psi^{*}\left(A_{S}\right)$ is an ideal in $\mathfrak{S}(M(A))$. Since $A_{S}$ is weak* dense in $M(S)$ [11, p. 158], it follows from what was said above that the weak* closure of $\Psi^{*}\left(A_{S}\right)$ is $M_{S}(T)=\Psi^{*}(M(S))$. By virtue of the separate weak* continuity of the convolution in $M(T)$ (Lemma 3.2), $M_{S}$ is therefore an ideal in $M(T)$, which contains $\mathfrak{S C}(M(A))$ as a weak* dense subspace. Thus (d) is proved. Since multiplication in $T$ corresponds to the convolution of Dirac measures, (b) is an immediate consequence of (d). Finally, (c) follows from the equation $\varphi(\psi \circ \varphi(t))=\varphi(t \psi(u))$, i.e., $\varphi(t)=\varphi(t) u$, since $\varphi$ is injective on $\psi(S)$ and $t \psi(u) \in \psi(S)$.

EXAMPLES. The above theorem is applicable e.g. in two classical situations, where the algebra $A$ is defined in terms of a locally compact abelian topological group $G$. If $A$ is $L^{1}(G)$, the convolution algebra of Haar integrable functions on $G$, then as is well known [5, p. 3] the multiplier algebra $M(A)$ may be identified with the convolution algebra $M(G)$ of bounded regular Borel measures on $G$. In this case, $S$ is the Bohr compactification of $G$ and $\psi(S)$ is the kernel (i.e., minimal ideal) of $T$ [11, p. 164].

Similarly, the theorem yields a connection between the structure semigroups of the convolution measure algebras

$$
L^{1}\left(G_{+}\right)=\left\{f \in L^{1}(G)\left|\int_{G \backslash G_{+}}\right| f(x) \mid d x=0\right\}
$$

and

$$
M\left(G_{+}\right)=\left\{\mu \in M(G)|| \mu \mid\left(G \backslash G_{+}\right)=0\right\},
$$

where $G_{+}$is a closed subsemigroup of $G$ containing the neutral element of $G$ and such that the interior of $G_{+}$generates $G$ and is dense in $G_{+}$. For $A=L^{1}\left(G_{+}\right)$satisfies the hypotheses of the theorem (for example, $S$ has an identity, since $G_{+}$may be realixed as a dense subsemigroup of $S$ [11, p. 163]), and Birtel has shown in [1, p. 821] that $M(A)=M\left(G_{+}\right)$. In the case of $A=L^{1}\left(G_{+}\right)$(and hence if $A=$ $L^{1}(G)$ ) the usual order in $M(A)$ as a space of measures agrees with the order defined before Theorem 3.1, for it follows from Birtel's proof that there is a net $\left\{\mu_{\sigma}\right\}$ of positive $\mu_{\sigma} \in L^{1}\left(G_{+}\right)$satisfying $\lim _{\sigma} \mu_{\sigma} * \mu(f)=\mu(f)$ for all $f \in C_{0}(G), \mu \in M\left(G_{+}\right)$.

\section{REFERENCES}

1. F. T. Birtel, On a commutative extension of a Banach algebra, Proc. Amer. Math. Soc., 13 (1962), 815-822.

2. N. Dunford and J. T. Schwartz, Linear Operators (Part I), Interscience, New York, 1958.

3. I. Glicksberg, Weak compactness and separate continuity, Pacific J. Math., 11 (1961), 205-214. 
4. E. Hewitt and K. A. Ross, Abstract Harmonic Analysis I, Springer, Berlin-GöttingenHeidelberg, 1963.

5. R. Larsen, An Introduction to the Theory of Multipliers, Springer, Berlin-HeidelbergNew York, 1971.

6. J. S. Pym, Dual structures for measure algebras, Proc. London Math. Soc., (3) 19 (1969), 625-660.

7. D. E. Ramirez, The measure algebra as an operator algebra, Canad. J. Math., 20 (1968), 1391-1396.

8. J. F. Rennison, Arens products and measure algebras, J. London Math. Soc., 44 (1969), 369-377.

9. M. A. Rieffel, A characterization of commutative group algebras and measure algebras, Trans. Amer. Math. Soc., 116 (1965), 32-65.

10. S. Sakai, $C^{*}$-algebras and $W^{*}$-algebras, Springer, Berlin-Heidelberg-New York, 1971.

11. J. L. Taylor, The structure of convolution measure algebras, Trans. Amer. Math. Soc., 119 (1965), 150-166.

12. - Convolution measure algebras with group maximal ideal spaces, Trans. Amer. Math. Soc., 128 (1967), 257-263.

13. K. Ylinen, The maximal ideal space of a Banach algebra of multipliers, Math. Scand., 27 (1970), 166-180.

Received November 8, 1972 and in revised form June 18, 1973.

University of Helsinki, Helsinki, Finland 



\section{PACIFIC JOURNAL OF MATHEMATICS}

\section{EDITORS}

RICHARD ARENS (Managing Editor)

University of California

Los Angeles, California 90024

R. A. BeAumont

University of Washington

Seattle, Washington 98105
J. DugundJI*

Department of Mathematics

University of Southern California

Los Angeles, California 90007

D. Gilbarg and J. Milgram

Stanford University

Stanford, California 94305

\section{ASSOCIATE EDITORS}

E. F. BECKENBACH

B. H. NEUMANN

F. WOLF

K. YoSHIDA

\section{SUPPORTING INSTITUTIONS}

UNIVERSITY OF BRITISH COLUMBIA
CALIFORNIA INSTITUTE OF TECHNOLOGY
UNIVERSITY OF CALIFORNIA
MONTANA STATE UNIVERSITY
UNIVERSITY OF NEVADA
NEW MEXICO STATE UNIVERSITY
OREGON STATE UNIVERSITY
UNIVERSITY OF OREGON
OSAKA UNIVERSITY

UNIVERSITY OF BRITISH COLUMBIA CALIFORNIA INSTITUTE OF TECHNOLOGY UNIVERSITY OF CALIFORNIA MONTANA STATE UNIVERSITY NEW MEXICO STATE UNIVERSITY UNIVERSITY OF OREGON OSAKA UNIVERSITY
UNIVERSITY OF SOUTHERN CALIFORNIA STANFORD UNIVERSITY UNIVERSITY OF TOKYO UNIVERSITY OF UTAH WASHINGTON STATE UNIVERSITY UNIVERSITY OF WASHINGTON AMERICAN MATHEMATICAL SOCIETY NAVAL WEAPONS CENTER

* C. R. DePrima California Institute of Technology, Pasadena, CA 91109, will replace J. Dugundji until August 1974. 


\section{Pacific Journal of Mathematics}

\section{Vol. 51, No. $1 \quad$ November, 1974}

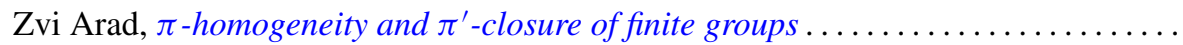

Ivan Baggs, A connected Hausdorff space which is not contained in a maximal

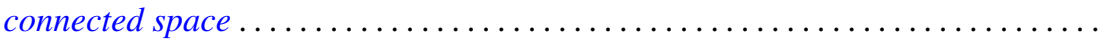

Eric Bedford, The Dirichlet problem for some overdetermined systems on the unit ball in $C^{n}$

R. H. Bing, Woodrow Wilson Bledsoe and R. Daniel Mauldin, Sets generated by

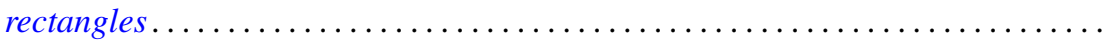

Carlo Cecchini and Alessandro Figà-Talamanca, Projections of uniqueness for

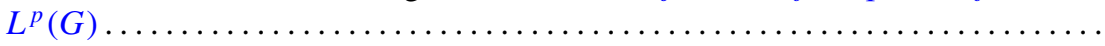

Gokulananda Das and Ram N. Mohapatra, The non absolute Nörlund summability of Fourier series .

Frank Rimi DeMeyer, On separable polynomials over a commutative ring ........ Richard Detmer, Sets which are tame in arcs in $E^{3} \ldots \ldots \ldots \ldots \ldots \ldots \ldots \ldots$

William Erb Dietrich, Ideals in convolution algebras on Abelian groups ..........

Bryce L. Elkins, A Galois theory for linear topological rings .................

William Alan Feldman, A characterization of the topology of compact convergence on $C(X)$.

Hillel Halkin Gershenson, A problem in compact Lie groups and framed cobordism

Samuel R. Gordon, Associators in simple algebras.

Marvin J. Greenberg, Strictly local solutions of Diophantine equations

Jon Craig Helton, Product integrals and inverses in normed rings . . . . . . . . . . . .

Domingo Antonio Herrero, Inner functions under uniform topology . . .

Jerry Alan Johnson, Lipschitz spaces .

Marvin Stanford Keener, Oscillatory solutions and multi-point boundary value

functions for certain nth-order linear ordinary differential equations.

John Cronan Kieffer, A simple proof of the Moy-Perez generalization of the

Shannon-McMillan theorem .......................

Joong Ho Kim, Power invariant rings

Gangaram S. Ladde and V. Lakshmikantham, On flow-invariant sets .

Roger T. Lewis, Oscillation and nonoscillation criteria for some self-adjoint even

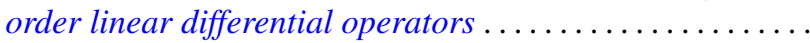

Jürg Thomas Marti, On the existence of support points of solid convex sets ..

John Rowlay Martin, Determining knot types from diagrams of knots . .

James Jerome Metzger, Local ideals in a topological algebra of entire functions

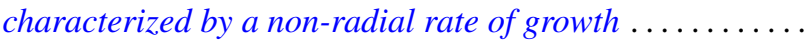

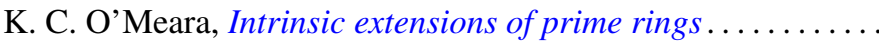

Stanley Poreda, A note on the continuity of best polynomial approximations ..

Robert John Sacker, Asymptotic approach to periodic orbits and local prolongations

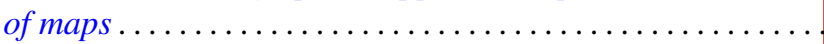

Eric Peter Smith, The Garabedian function of an arbitrary compact set . .

Arne Stray, Pointwise bounded approximation by functions satisfying a side condition

John St. Clair Werth, Jr., Maximal pure subgroups of torsion complete abelian

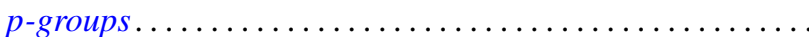

